Journal of Mathematics and Informatics

Vol. 10, 2017, 75-81

ISSN: 2349-0632 (P), 2349-0640 (online)

Published 11 December 2017

www.researchmathsci.org

DOI: http://dx.doi.org/10.22457/jmi.v10a10

Journal of

Mathematics and

Informatics

\title{
On the Binary Quadratic Diophantine Equation
}

\author{
$y^{2}=80 x^{2}-16$ \\ M.Devi ${ }^{1}$ and T.R.Usha Rani ${ }^{2}$
}

${ }^{1}$ Department of Mathematics, Shrimati Indira Gandhi College, Trichy-620002,

Tamilnadu, India. e-mail: swethadev12345@gmail.com

${ }^{2}$ Department of Mathematics, Shrimati Indira Gandhi College, Trichy-620002,

Tamilnadu,India.e-mail: usharanisigc@gmail.com

Received 15 November 2017; accepted 4 December 2017

Abstract. The Binary quadratic negative pell equation $y^{2}=80 x^{2}-16$ representing a hyperbola is analyzed for its non-zero integer solutions. A few interesting relations among its solutions are presented. Further, employing the solutions of the above equation, we have obtained solutions of other choices of hyperbolas, parabolas and special pythogorean triangles.

Keywords: Binary quadratic, hyperbola, parabola, negative pell equation, integral solutions.

\section{AMS Mathematics Subject Classification (2010): 11D09}

\section{Introduction}

The binary quadratic diophantine equations (both homogeneous and non-homogeneous) are rich in variety. In [1-8] the binary quadratic non-homogeneous equations representing hyperbolas respectively are studied for their non-zero integral solutions. This communication concerns with yet another binary quadratic equation given by $y^{2}=80 x^{2}-16$. The recurrence relations satisfied by the solutions $x$ and $y$ are given. Also a few interesting properties among the solutions are exhibited. Further, employing the solutions of the above hyperbola, we have obtained solutions of other choices of hyperbolas, parabolas and special pythogorean triangles.

\section{Method of analysis:}

The negative pell equation representing hyperbola under consideration is

$$
y^{2}=80 x^{2}-16
$$

whose smallest positive integer solution is $x_{0}=1, y_{0}=8$.

To obtain the other solutions of (1), consider the pell equation $y^{2}=80 x^{2}-16$ whose solution is given by

$$
\begin{aligned}
& \tilde{x}_{n}=\frac{1}{2 \sqrt{80}} g_{n} \\
& \tilde{y}_{n}=\frac{1}{2} f_{n}
\end{aligned}
$$




\section{M.Devi and T.R.Usha Rani}

where,

$$
\begin{aligned}
& f_{n}=(9+\sqrt{80})^{n+1}+(9-\sqrt{80})^{n+1} \\
& g_{n}=(9+\sqrt{80})^{n+1}-(9-\sqrt{80})^{n+1}
\end{aligned}
$$

Applying Brahmagupta lemma between the solutions $\left(x_{0}, y_{0}\right)$ and $\left(x_{n}, y_{n}\right)$, the other integer solutions of (1) are given by

$$
\begin{aligned}
& x_{n+1}=\frac{1}{2} f_{n}+\frac{8}{2 \sqrt{80}} g_{n}, \\
& y_{n+1}=4 f_{n}+\frac{40}{\sqrt{80}} g_{n}, \text { where } n=-1,0,1, \ldots .
\end{aligned}
$$

Recurrence relations for $x$ and $y$ are:

$$
x_{n+3}-18 x_{n+2}+x_{n+1}=0
$$

$y_{n+3}-18 y_{n+2}+y_{n+1}=0$, where $n=-1,0,1, \ldots$.

Some numerical examples of $x$ and $y$ satisfying (1) are given in the Table 1 below:

Table 1: Examples

\begin{tabular}{|l|l|l|}
\hline$n$ & $x_{n}$ & $y_{n}$ \\
\hline-1 & 1 & 8 \\
\hline 0 & 17 & 152 \\
\hline 1 & 305 & 2728 \\
\hline 2 & 5473 & 48952 \\
\hline
\end{tabular}

From the above table, we observe some interesting relations among the solutions which are presented below.

$>$ The values of $x_{n}$ and $y_{n}$ are odd and even respectively.

Each of the following expressions is a nasty number.

- $\frac{1}{16}\left[192+1824 x_{2 n+2}-96 x_{2 n+3}\right]$

- $\frac{1}{288}\left[3456+32736 x_{2 n+2}-96 x_{2 n+4}\right]$

- $\frac{1}{16}\left[192+960 x_{2 n+2}-96 y_{2 n+2}\right]$

- $\frac{1}{144}\left[1728+16320 x_{2 n+2}-96 y_{2 n+3}\right]$

- $\frac{1}{2576}\left[30912+292800 x_{2 n+2}-96 y_{2 n+4}\right]$

- $\frac{1}{16}\left[192+32736 x_{2 n+3}-1824 x_{2 n+4}\right]$

- $\frac{1}{144}\left[1728+960 x_{2 n+3}-1824 y_{2 n+2}\right]$ 
On the Binary Quadratic Diophantine Equation $y^{2}=80 x^{2}-16$

- $\frac{1}{16}\left[192+16320 x_{2 n+3}-1824 y_{2 n+3}\right]$

- $\frac{1}{144}\left[1728+292800 x_{2 n+3}-1824 y_{2 n+4}\right]$

- $\frac{1}{2576}\left[30912+960 x_{2 n+4}-32736 y_{2 n+2}\right]$

- $\frac{1}{144}\left[1728+16320 x_{2 n+4}-32736 y_{2 n+3}\right]$

- $\frac{1}{16}\left[192+292800 x_{2 n+4}-32736 y_{2 n+4}\right]$

- $\frac{1}{1280}\left[15360+960 y_{2 n+3}-16320 y_{2 n+2}\right]$

- $\frac{1}{23040}\left[276480+960 y_{2 n+4}-292800 y_{2 n+2}\right]$

- $\frac{1}{1280}\left[15360+16320 y_{2 n+4}-292800 y_{2 n+3}\right]$

Each of the following expressions is a cubical integer.

- $\frac{1}{16}\left[\left(304 x_{3 n+3}-16 x_{3 n+4}\right)+3\left(304 x_{n+1}-16 x_{n+2}\right)\right]$

- $\frac{1}{288}\left[\left(5456 x_{3 n+3}-16 x_{3 n+5}\right)+3\left(5456 x_{n+1}-16 x_{n+3}\right)\right]$

- $\frac{1}{16}\left[\left(160 x_{3 n+3}-16 y_{3 n+3}\right)+3\left(160 x_{n+1}-16 y_{n+1}\right)\right]$

- $\frac{1}{144}\left[\left(2720 x_{3 n+3}-16 y_{3 n+4}\right)+3\left(2720 x_{n+1}-16 y_{n+2}\right)\right]$

- $\frac{1}{2576}\left[\left(48800 x_{3 n+3}-16 y_{3 n+5}\right)+3\left(48800 x_{n+1}-16 y_{n+3}\right)\right]$

- $\frac{1}{16}\left[\left(5456 x_{3 n+4}-304 x_{3 n+5}\right)+3\left(5456 x_{n+2}-304 x_{n+3}\right)\right]$

- $\frac{1}{144}\left[\left(160 x_{3 n+4}-304 y_{3 n+3}\right)+3\left(160 x_{n+2}-304 y_{n+1}\right)\right]$

- $\frac{1}{16}\left[\left(2720 x_{3 n+4}-304 y_{3 n+4}\right)+3\left(2720 x_{n+2}-304 y_{n+2}\right)\right]$

- $\frac{1}{144}\left[\left(48800 x_{3 n+4}-304 y_{3 n+5}\right)+3\left(48800 x_{n+2}-304 y_{n+3}\right)\right]$

- $\frac{1}{2576}\left[\left(160 x_{3 n+5}-5456 y_{3 n+3}\right)+3\left(160 x_{n+3}-5456 y_{n+1}\right)\right]$

- $\frac{1}{144}\left[\left(2720 x_{3 n+5}-5456 y_{3 n+4}\right)+3\left(2720 x_{n+3}-5456 y_{n+2}\right)\right]$

- $\frac{1}{16}\left[\left(48800 x_{3 n+5}-5456 y_{3 n+5}\right)+3\left(48800 x_{n+3}-5456 y_{n+3}\right)\right]$ 


\section{M.Devi and T.R.Usha Rani}

- $\frac{1}{1280}\left[\left(160 y_{3 n+4}-2720 y_{3 n+3}\right)+3\left(160 y_{n+2}-2720 y_{n+1}\right)\right]$

- $\frac{1}{23040}\left[\left(160 y_{3 n+5}-48800 y_{3 n+3}\right)+3\left(160 y_{n+3}-48800 y_{n+1}\right)\right]$

- $\frac{1}{1280}\left[\left(2720 y_{3 n+5}-48800 y_{3 n+4}\right)+3\left(2720 y_{n+3}-48800 y_{n+2}\right)\right]$

Relations among the solutions :

- $x_{n+1}-18 x_{n+2}+x_{n+3}=0$

- $9 x_{n+1}-x_{n+2}+y_{n+1}=0$

- $9 x_{n+3}-161 x_{n+2}-y_{n+1}=0$

- $x_{n+1}-9 x_{n+2}+y_{n+2}=0$

- $y_{n+1}+80 x_{n+2}-9 y_{n+2}=0$

- $9 x_{n+1}-161 x_{n+2}+y_{n+3}=0$

- $9 x_{n+3}-x_{n+2}-y_{n+3}=0$

- $80 x_{n+1}-y_{n+2}+9 y_{n+1}=0$

- $1440 x_{n+1}-y_{n+3}+161 y_{n+1}=0$

- $160 x_{n+2}-y_{n+3}+y_{n+1}=0$

- $80 x_{n+2}-y_{n+3}+9 y_{n+2}=0$

- $y_{n+1}-18 y_{n+2}+y_{n+3}=0$

- $80 x_{n+1}-9 y_{n+3}+161 y_{n+2}=0$

- $161 x_{n+1}-x_{n+3}+18 y_{n+1}=0$

- $x_{n+1}-x_{n+3}-2 y_{n+2}=0$

- $9 x_{n+2}-x_{n+3}+y_{n+2}=0$

- $288 y_{n+1}+2560 x_{n+3}-5152 y_{n+2}=0$

- $x_{n+1}-161 x_{n+3}+18 y_{n+3}=0$

- $y_{n+1}+1440 x_{n+3}-161 y_{n+3}=0$

- $y_{n+2}+80 x_{n+3}-9 y_{n+3}=0$

\section{Remarkable observations}

3.1. Employing linear combinations among the solutions of (1), one may generate integer solutions for other choices of hyperbola which are presented in Table 2 below. 
On the Binary Quadratic Diophantine Equation $y^{2}=80 x^{2}-16$

Table 2: Hyperbola

\begin{tabular}{|c|c|c|}
\hline S.No & Hyperbola & $\left(X_{n}, Y_{n}\right)$ \\
\hline 1 & $80 X_{n}^{2}-Y_{n}^{2}=81920$ & {$\left[\left(304 x_{n+1}-16 x_{n+2}\right),\left(160 x_{n+2}-2720 x_{n+1}\right)\right]$} \\
\hline 2 & $80 X_{n}^{2}-Y_{n}^{2}=26542080$ & {$\left[\left(5456 x_{n+1}-16 x_{n+3}\right),\left(160 x_{n+3}-48800 x_{n+1}\right)\right]$} \\
\hline 3 & $80 X_{n}^{2}-Y_{n}^{2}=81920$ & {$\left[\left(160 x_{n+1}-16 y_{n+1}\right),\left(160 y_{n+1}-1280 x_{n+1}\right)\right]$} \\
\hline 4 & $80 X_{n}^{2}-Y_{n}^{2}=6635520$ & {$\left[\left(2720 x_{n+1}-16 y_{n+2}\right),\left(160 y_{k+2}-24320 x_{n+1}\right)\right]$} \\
\hline 5 & $80 X_{n}^{2}-Y_{n}^{2}=2123448320$ & {$\left[\left(48800 x_{n+1}-16 y_{n+3}\right),\left(160 y_{n+3}-436480 x_{n+1}\right)\right]$} \\
\hline 6 & $80 X_{n}^{2}-Y_{n}^{2}=81920$ & {$\left[\left(5456 x_{n+2}-304 x_{z+3}\right),\left(2720 x_{n+3}-48800 x_{n+2}\right)\right]$} \\
\hline 7 & $80 X_{n}^{2}-Y_{n}^{2}=6635520$ & {$\left[\left(160 x_{n+2}-304 y_{n+1}\right),\left(2720 y_{n+1}-1280 x_{n+2}\right)\right]$} \\
\hline 8 & $80 X_{n}^{2}-Y_{n}^{2}=81920$ & {$\left[\left(2720 x_{n+2}-304 y_{*+2}\right) \cdot\left(2720 y_{n+2}-24320 x_{n+2}\right)\right]$} \\
\hline 9 & $80 X_{n}^{2}-Y_{n}^{2}=6635520$ & {$\left[\left(48800 x_{n+2}-304 y_{n+3}\right),\left(2720 y_{n+3}-436480 x_{n+2}\right)\right]$} \\
\hline 10 & $80 X_{n}^{2}-Y_{n}^{2}=2123448320$ & {$\left[\left(160 x_{n+3}-5456 y_{n+1}\right),\left(48800 y_{n+1}-1280 x_{n+3}\right)\right]$} \\
\hline 11 & $80 X_{n}^{2}-Y_{n}^{2}=6635520$ & {$\left[\left(2720 x_{n+3}-5456 y_{k+2}\right),\left(48800 y_{k+2}-24320 x_{n+3}\right)\right]$} \\
\hline 12 & $80 X_{n}^{2}-Y_{n}^{2}=81920$ & {$\left[\left(48800 x_{n+3}-5456 y_{n+3}\right),\left(48800 y_{n+3}-436480 x_{n+3}\right)\right]$} \\
\hline 13 & $80 X_{n}^{2}-Y_{n}^{2}=52428800$ & {$\left[\left(160 y_{n+2}-2720 y_{n+1}\right),\left(24320 y_{k+1}-1280 y_{n+2}\right)\right]$} \\
\hline 14 & $80 X_{n}^{2}-Y_{n}^{2}=169869312000$ & {$\left[\left(160 y_{n+3}-48800 y_{n+1}\right),\left(436480 y_{n+1}-1280 y_{n+3}\right)\right]$} \\
\hline 15 & $80 X_{n}^{2}-Y_{n}^{2}=524288000$ & {$\left[\left(2720 y_{n+3}-48800 y_{n+2}\right),\left(436480 y_{k+2}-24320 y_{n+3}\right)\right]$} \\
\hline
\end{tabular}

3.2. Employing linear combinations among the solutions of (1), one may generate integer solutions for other choices of parabola which are presented in Table 3 below. 


\section{M.Devi and T.R.Usha Rani}

Table 3: Parabola

\begin{tabular}{|c|c|c|}
\hline $\begin{array}{l}\text { S. } \\
\text { No }\end{array}$ & Parabola & $\left(X_{n}, Y_{*}\right)$ \\
\hline 1 & $1280 X_{n}-Y_{n}^{2}=\$ 1920$ & {$\left[\left(32+304 x_{2 n+2}-16 x_{2 n+3}\right),\left(160 x_{n+2}-2720 x_{n+1}\right)\right]$} \\
\hline 2 & $23040 X_{n}-Y_{n}^{2}=26542080$ & {$\left[\left(576+5456 x_{2 n+2}-16 x_{2 n+4}\right)_{0}\left(160 x_{n+3}-48800 x_{n+1}\right)\right]$} \\
\hline 3 & $1280 X_{n}-Y_{n}^{2}=81920$ & {$\left[\left(32+160 x_{2 k+2}-16 y_{2 k+2}\right),\left(160 y_{k+1}-1280 x_{n+1}\right)\right]$} \\
\hline 4 & $11520 X_{n}-Y_{n}^{2}=6635520$ & {$\left[\left(288+2720 x_{2 n+2}-16 y_{2 n+3}\right),\left(160 y_{n+2}-24320 x_{n+1}\right)\right]$} \\
\hline 5 & $206080 X_{n}-Y_{n}^{2}=2123448320$ & {$\left[\left(5152+48800 x_{2 n+2}-16 y_{2 n+4}\right),\left(160 y_{n+3}-436480 x_{n+1}\right)\right]$} \\
\hline 6 & $1280 X_{n}-Y_{n}^{2}=81920$ & {$\left[\left(32+5456 x_{2 n+3}-304 x_{2 n+4}\right),\left(2720 x_{n+3}-48800 x_{n+2}\right)\right]$} \\
\hline 7 & $11520 X_{n}-Y_{n}^{2}=6635520$ & {$\left[\left(288+160 x_{2 n+3}-304 y_{2 n+2}\right)_{0}\left(2720 y_{n+1}-1280 x_{n+2}\right)\right]$} \\
\hline 8 & $1280 X_{n}-Y_{n}^{2}=81920$ & {$\left[\left(32+2720 x_{2 n+3}-304 y_{2 n+3}\right),\left(2720 y_{n+2}-24320 x_{n+2}\right)\right]$} \\
\hline 9 & $11520 X_{n}-Y_{n}^{2}=6635520$ & {$\left[\left(288+48800 x_{2 n+3}-304 y_{2 n+4}\right),\left(2720 y_{n+3}-436480 x_{n+2}\right)\right]$} \\
\hline 10 & $206080 X_{n}-Y_{n}^{2}=2123448320$ & {$\left[\left(2254+160 x_{2 n+4}-5456 y_{2 n+2}\right)\left(48800 y_{n+1}-1280 x_{n+3}\right)\right]$} \\
\hline 11 & $11520 X_{n}-Y_{n}^{2}=6635520$ & {$\left[\left(230+2720 x_{2 n+4}-5456 y_{2 *+3}\right),\left(48800 y_{n+2}-24320 x_{n+3}\right)\right]$} \\
\hline 12 & $1280 X_{n}-Y_{n}^{2}=81920$ & {$\left[\left(32+48800 x_{2 n+4}-5456 y_{2 n+4}\right),\left(48800 y_{n+3}-436480 x_{n+3}\right)\right]$} \\
\hline 13 & $102400 X_{n}-Y_{n}^{2}=52428800$ & {$\left[2560+\left(160 y_{2 n+3}-2720 y_{2 s+2}\right),\left(24320 y_{n+1}-1280 y_{n+2}\right)\right]$} \\
\hline 14 & $1843200 X_{n}-Y_{n}^{2}=169869312000$ & {$\left[\left(46080+160 y_{2 n+4}-48800 y_{2 n+2}\right)_{*}\left(436480 y_{n+1}-1280 y_{k+3}\right)\right]$} \\
\hline 15 & $102400 X_{n}-Y_{n}^{2}=524288000$ & {$\left[\left(2560+2720 y_{2 n+4}-48800 y_{2 n+3}\right),\left(436480 y_{n+2}-24320 y_{n+3}\right.\right.$} \\
\hline
\end{tabular}

3.3. Consider $m=x_{n+1}+y_{n+1}, n=x_{n+1}$, observe that $m>n>0$.

Treat $m, n$ as the generators of the pythogorean triangle $\mathrm{T}(\alpha, \beta, \gamma)$,

$$
\alpha=2 m n, \beta=m^{2}-n^{2}, \gamma=m^{2}+n^{2} .
$$

Then the following interesting relations are observed.
1) $\alpha-40 \beta+39 \gamma=16$
2) $41 \alpha-\gamma-\frac{160 A}{P}=16$
3) $21 \alpha-20 \beta+19 \gamma-\frac{80 A}{P}=16$
4) $\frac{2 A}{P}=x_{n+1} y_{n+1}$ 
On the Binary Quadratic Diophantine Equation $y^{2}=80 x^{2}-16$

\section{Conclusion}

In this paper, we have presented infinitely many integer solutions for all hyperbola represented by the negative pellequation $y^{2}=80 x^{2}-16$. As the binary quadratic Diophantine equations are rich in variety, one may search for the other choices of equations and determine their integer solutions along with suitable properties.

\section{REFERENCES}

1. R.A.Mollin and A. Srinivasan, A note on the negative pell equation, International Journal of Algebra, 4(19) (2010) 919-922.

2. E.E.Whitford, Some solutions of the Pellian equation $x^{2}-A y^{2}= \pm 4$, Annals of Mathematics, 1 (1913-1914) 157-160.

3. S.Ahmet Tekcan, Bet W Gezer and Osman Bizim, On the integer solutions of the pellEquations $x^{2}-d y^{2}=2^{t}$, World Academy of Science, Engineering and Technology, 1 (2007) 522-526.

4. S.Ahmet Tekcan, The pell equation $x^{2}-\left(k^{2}-k\right) y^{2}=2^{t}$, World Academy of Science, Engineering and Technology, 19 (2008) 697-701.

5. V.Sangeetha, M.A.Gopalan and M.Somanath, On the integral solutions of the pell equation $x^{2}=13 y^{2}-3^{t}$, International Journal of Applied Mathematical Research, 3(1) (2014) 58-61.

6. M.A.Gopalan, G.Sumathi and S.Vidhyalakshmi, Observations on the hyperbola $x^{2}=19 y^{2}-3^{t}$, Journal of the Engineering and Technology, 2(2A) (2014) 152-155.

7. M.A.Gopalan,S.Vidhyalakshmi and A. Kavitha, On the integral solutions of the binary quadratic equation $x^{2}=15 y^{2}-11^{t}$, Scholars Journal of the Engineering and Technology, 2(2A) (2014) 156-158.

8. S.Vidhyalakshmi, V.Krithika and K.Agalya, On the negative pell equation, Proceeding of the National Conference on MATAM, (2015) 4-9. 